\title{
Endovascular Repair of Descending Thoracic Aneurysms: Results With "On-Label" Application in the Post Food and Drug Administration Approval Era
}

\author{
G. Chad Hughes, MD, Sean M. Lee, MD, Mani A. Daneshmand, MD, Syamal D. \\ Bhattacharya, MD, Judson B. Williams, MD, Sonny W. Tucker Jr, MD, and Richard L. \\ McCann, MD \\ Department of Surgery, Duke University Medical Center, Durham, North Carolina
}

\section{Abstract}

\begin{abstract}
Background-Most studies of thoracic endovascular aortic repair (TEVAR) published since the technology gained US Food and Drug Administration (FDA) approval in March 2005 have included multiple applications including dissection, trauma, and "hybrid" approaches, all of which are currently "off-label." However, little post-approval data exist for the only FDA-approved application, namely descending thoracic aneurysm (DTA). The purpose of this study was to examine our experience with TEVAR for aneurysms limited to the descending thoracic aorta.
\end{abstract}

Methods-Between March 23, 2005 (date of initial FDA approval) and April 6, 2009, 210 TEVAR procedures were performed at our institution. Of these, $79(38 \%)$ were for saccular $(\mathrm{n}=$ $31)$ or fusiform ( $n=48)$ DTA and form the basis of this report. Patients requiring "hybrid" approaches other than carotid-subclavian bypass were excluded. Devices utilized were Gore TAG (W. L. Gore Associates, Flagstaff, AZ) $(\mathrm{n}=67 ; 85 \%)$, Zenith TX2 (Cook Medical Incorporated, Bloomington, IN) ( $\mathrm{n}=10 ; 13 \%)$, and Medtronic Talent (Medtronic, Inc, Santa Rosa, CA) ( $\mathrm{n}=5$; $6 \%) ; 3(4 \%)$ patients received more than one type of device.

Results-Median patient age was $73 \pm 4$ years; 35 (44\%) were female. Mean aortic diameter was $5.8 \pm 1.8 \mathrm{~cm}$. Twenty-four (30\%) procedures were urgent-emergent. Thirty-day inhospital rates of death, stroke, and permanent paraplegia-paresis were $5.1 \%(\mathrm{n}=4 ; 1.9 \%$ elective mortality), $2.5 \%$ $(\mathrm{n}=2)$, and $1.3 \%(\mathrm{n}=1)$, respectively. The median postoperative length of stay was 3.0 days (25th and 75th percentiles $=2$ and 6 , respectively). At a mean follow-up of $23 \pm 17$ months (range, 6 to $55)$, there were $2(2.5 \%)$ late aortic deaths from graft infection $(\mathrm{n}=1)$ and aneurysm rupture $(\mathrm{n}=$ 1). Overall actuarial midterm survival is $73 \%$ at 55 months, with an aorta-specific actuarial survival of $86 \%$ during this same time interval. Five patients $(6.3 \%)$ required late ( $>30$ days) secondary endovascular re-intervention for type I $(n=4)$ or type II $(n=1)$ endoleak; reintervention was successful in 4 of 5 .

Conclusions-Despite the advanced age, comorbid conditions, and significant incidence of urgent-emergent status of patients presenting with DTA, on-label application of TEVAR yields excellent 30-day and midterm outcomes, especially when compared with historic rates of morbidity and mortality with open repair. However, "on-label" applications represent a minority of current TEVAR use, likely due to the relative scarcity of DTA. These data appear to support the increasing utilization of TEVAR as a treatment strategy for this pathology. 
On March 23, 2005, the US Food and Drug Administration (FDA) granted approval for the first endovascular device specifically designed for the treatment of aneurysms of the descending thoracic aorta. Food and Drug approval was based largely on the results of two clinical trials of highly selected patients with descending thoracic aneurysms [1, 2]. Most studies of thoracic endovascular aortic repair (TEVAR) published since the technology gained FDA approval have included multiple applications including dissection, trauma, and "hybrid" approaches, all of which are currently "off-label" [3]. However, little post-approval data exist for the only FDA-approved application, namely repair of descending thoracic aneurysms. The purpose of this study was to examine our experience with TEVAR for aneurysms limited to the descending thoracic aorta.

\section{Patients and Methods}

Between March 23, 2005 (date of FDA approval of the first available thoracic device in the US) and April 6, 2009, 210 TEVAR procedures were performed at our institution. Of these, $79(38 \%)$ were for saccular $(n=31)$ or fusiform $(n=48)$ descending thoracic aneurysms and form the basis of this report. All landing zones were between the left common carotid artery and celiac axis. Patients requiring "hybrid" approaches [4] other than carotid-subclavian bypass were excluded. Criteria for repair included symptoms, rapid enlargement $(>5 \mathrm{~mm}$ in 12 months), or absolute size. For fusiform aneurysms, this generally included a diameter of $5.5 \mathrm{~cm}$ or greater, whereas for saccular aneurysms a protrusion of $2 \mathrm{~cm}$ or greater beyond the aortic wall for the saccular component was considered an indication for treatment in the absence of symptoms [5]. Emergent cases were defined as those requiring immediate operative therapy within 24 hours of admission; all emergent cases in the present series were ruptured aneurysms. Urgent cases were defined by intact, acutely symptomatic aneurysms requiring operative intervention within the same hospitalization. The presence of a connective tissue disorder such as Marfan or Loeys-Dietz syndrome was considered a contraindication to TEVAR [6]. The study was approved by the Duke Institutional Review Board (IRB) and the IRB waived the need for individual patient consent.

Devices utilized were Gore TAG (W.L Gore \& Associates, Flagstaff, AZ) $(n=67 ; 85 \%)$, Zenith TX2 (Cook Medical Incorporated, Bloomington, IN) $(\mathrm{n}=10 ; 13 \%)$, and Medtronic Talent (Medtronic, Inc, Santa Rosa, CA) $(\mathrm{n}=5 ; 6 \%) ; 3(4 \%)$ patients received more than one type of device. Fifteen patients (19\%) required an iliac conduit to allow safe introduction of the introducer sheath necessary for the procedure. The left subclavian artery was partially or fully covered in 33 patients (42\%), of whom 4 (12\% of those in whom the left subclavian was covered) underwent adjunctive left carotid-subclavian bypass during the same operation as endovascular repair. Indications for left carotid-subclavian bypass were as previously described [3].

Preoperative planning of endograft procedures was performed using the TeraRecon system (TeraRecon Inc, San Mateo, CA), which allows highly accurate centerline measurements of flow lumen diameter to assess landing zones as well as iliofemoral access vessels. Intraoperative intravascular ultrasound using the Volcano system (Volcano Corporation, San Diego, CA) was used selectively $(\mathrm{n}=15 ; 19 \%)$ as needed, usually for confirmatory measurements of landing zone diameters or adequacy. All endovascular procedures were performed in the operating room under general anesthesia; adjunctive trans-esophageal echocardiography was used routinely.

On-line monitoring of spinal cord function with somatosensory and motor evoked potentials was used intraoperatively in elective cases and when available for urgent-emergent cases (n $=55$ cases monitored; 70\%) using previously described techniques [7]. Cerebrospinal fluid drainage was used selectively $(\mathrm{n}=14 ; 18 \%)$ for previously described indications [3]. 
Comorbidities were defined using standard definitions. All procedural outcomes and complications were prospectively recorded. Patient follow-up included clinical examination, four-view chest X-ray, and computed tomographic angiography at 1 month, 6 months, and 12 months postoperatively and annually thereafter. In addition, 3-month follow-up assessment and imaging was obtained in patients with an endoleak identified at 1 month, if the decision for initial endoleak observation was made. Noncontrasted magnetic resonance imaging, in lieu of contrast-enhanced computed tomographic angiography, was obtained in patients with serum creatinine greater than 2.0. All follow-up was done at the Duke University Center for Aortic Surgery. This report includes all data collected through the patients' most recent follow-up visit. In addition, the social security death index was queried (http://ssdi.rootsweb.com/) to confirm all patient deaths. For those patients dying in followup, cause of death was confirmed by review of medical records or family interview in all cases. Survival analyses were performed using the Kaplan-Meier method. All data are presented in accordance with the "Reporting standards for endovascular aortic aneurysm repair" of the Ad Hoc Committee for Standardized Reporting Practices in Vascular Surgery of The Society for Vascular Surgery/American Association for Vascular Surgery [8].

\section{Results}

\section{Patient Demographics}

Mean aortic diameter was $5.8 \pm 1.8 \mathrm{~cm}$. Patient demographics are presented in Table 1 . Thirty-two patients $(41 \%)$ had undergone prior aortic repair, including root-ascending $(\mathrm{n}=$ $9)$, abdominal ( $\mathrm{n}=15$ open, $\mathrm{n}=3$ endovascular), open thoracic $(\mathrm{n}=2)$, or open thoracoabdominal aortic repair $(n=3)$. Extent of aortic coverage by the endograft(s) is presented in Table 2. Sixty-three percent of patients had coverage of higher risk aorta between T6 and the celiac axis [9]. Twenty-four (30\%) cases were urgent $(\mathrm{n}=21 ; 27 \%)$ or emergent $(\mathrm{n}=3 ; 4 \%)$. Three patients (4\%) presented with aortic rupture, all of whom were taken emergently to the operating room and remained hemodynamically stable at the time of surgery.

\section{Procedural (30-Day) Outcomes}

The median number of stent grafts implanted per case was $2.0 \pm 0.7$ (range, 1 to 4). Primary technical success, defined as successful endograft deployment with secure proximal and distal fixation with no type I or III endoleak and absence of open surgical conversion or mortality within the first 24 hours postoperatively [8], was achieved in 78 of 79 cases $(98.7 \%)$. The single case in which primary technical success was not achieved has been detailed previously [3] and involved free aortic rupture several hours postoperatively in a patient initially presenting with contained rupture. Autopsy demonstrated inadequate distal seal zone. Two previously described patients [10] (2.5\%) underwent additional TEVAR during the index hospitalization for type I endoleaks discovered secondary to elevated aneurysm sac pulse pressure readings from an EndoSure wireless aneurysm sac pressure monitor (CardioMEMS Inc, Atlanta, GA) implanted at the time of TEVAR. Thus, a total of $3(3.8 \%)$ type I endoleaks were observed during the index hospitalization, 2 of 3 of which were successfully repaired prior to discharge.

Thirty-day in-hospital rates of death, stroke (defined as new neurologic deficit by clinical exam lasting greater than 24 hours, with or without computed tomographic-magnetic resonance imaging confirmation, or a finding on computed tomographic-magnetic resonance imaging of the brain consistent with an acute infarct), and permanent paraplegia-paresis were $5.1 \%(n=4 ; 1.9 \%$ elective and $12.5 \%$ urgent-emergent mortality), $2.5 \%(n=2)$, and $1.3 \%(\mathrm{n}=1)$, respectively. There were no posterior circulation or brain stem strokes related to intentional left subclavian artery coverage by the stent grafts. The single permanent spinal 
cord complication has been previously described [3] and recovered by two-year follow-up. An additional two patients developed some degree of delayed onset paraparesis during the postoperative period, both of which resolved completely with blood pressure augmentation, with or without cerebrospinal fluid drainage, and did not recur. Renal failure with new dialysis occurred in $2.5 \%(\mathrm{n}=2)$. There was a single $(1.3 \%)$ retrograde type A aortic dissection successfully repaired. One patient required Palmaz (Cordis Corporation, Miami Lakes, FL) bare metal stent re-expansion of partial proximal TAG graft collapse on postoperative day three [3]. The median postoperative length of stay was 3.0 days (25th and 75 th percentiles $=2$ and 6 , respectively).

\section{Follow-Up Outcomes}

Follow-up is $100 \%$ complete. Mean duration of follow-up is $23 \pm 17$ months (range, 6 to 55). Overall actuarial survival is $73 \%$ at 55 months, with an aorta-specific actuarial survival of $86 \%$ during this same time interval (Fig 1). Of the late deaths, $2(2.5 \%)$ were aortic related and the remainder $(n=14)$ were due to comorbid conditions. One aortic related death was due to persistent type I endoleak after failed re-intervention with subsequent aneurysm rupture and has been previously described [3]. The other aortic-related death was due to sepsis from an infected endograft 8 months postoperatively in an 80-year-old female who was not a candidate for open conversion. Interestingly, this patient had previously undergone open repair of an extent III thoracoabdominal aneurysm 18 years prior; that graft had become infected postoperatively and was treated definitively with antibiotic therapy. The antibiotics were discontinued after a number of years with no evidence for recurrent infection. This graft subsequently served as distal landing zone for TEVAR of a $7.5-\mathrm{cm}$ descending aneurysm developing above the area of prior repair. The TEVAR apparently reactivated the previously quiescent infection, which did not subsequently respond to antibiotic therapy.

The incidence of type I or III endoleak at the latest follow-up visit was $2.5 \%(\mathrm{n}=2)$. One patient suffered an aortic-related death as previously described [3] after an unsuccessful attempt at endovascular re-intervention; the other patient is an 87-year-old female who underwent initially successful urgent TEVAR for a $6.1-\mathrm{cm}$ saccular aneurysm with impending rupture. She developed a new distal type I endoleak on her two-year follow-up imaging at age 89 and refuses further endovascular intervention. Eight (10\%) patients had type II endoleaks, all due to intercostal arteries, noted at latest follow-up imaging. These are being followed with stable $(n=3)$ or decreasing $(n=5)$ aortic dimensions. Of those patients with follow-up imaging at 6 months or greater, $91 \%(n=42$ of 46$)$ have aortic dimensions which have decreased by at least $5 \mathrm{~mm}$.

The need for late (>30 days) re-intervention during follow-up is $6.3 \%(\mathrm{n}=5)$. This includes additional TEVAR for proximal type I endoleak in 3 at 1, 3, and 38 months postoperatively; visceral debranching plus TEVAR for a distal type I endoleak at 24 months postoperatively; and coil embolization of a type II endoleak from a left subclavian artery at 5 months postoperatively. Four of five of these procedures have been successful, with the only failure the previously described [3] patient dying of aortic rupture due to unrepaired proximal type I endoleak. Overall actuarial freedom from late re-intervention is $91 \%$ at 55 months (Fig 2).

\section{Comment}

Most studies of TEVAR published since the technology gained FDA approval in March 2005 have included multiple applications including dissection, trauma, and "hybrid" approaches, all of which are currently "off-label" [3]. However, little post-approval data exists for the only FDA-approved application, namely repair of descending thoracic aneurysms, likely due to the relative scarcity of descending aneurysm. The present study, 
which represents the largest report of TEVAR for degenerative aneurysms of the descending thoracic aorta treated in the post-FDA approval era, demonstrates that despite the advanced age, comorbid conditions, and significant incidence of nonelective status of patients presenting with descending aneurysm, on-label application of TEVAR yields excellent 30day and late midterm outcomes. The 30-day outcomes presented, namely an elective mortality rate of $1.9 \%$ with rates of stroke and permanent paraparesis-paraplegia of $2.5 \%$ and $1.3 \%$, respectively, are similar, if not superior, to those seen in the TEVAR clinical trial setting $[1,2]$ of highly selected descending aneurysm patients. For comparison, the larger of the two Gore TAG clinical trials [2] reported 30-day rates of death, stroke, and paraplegiaparesis of $2.1 \%, 3.6 \%$, and $2.9 \%$, respectively. Follow-up outcomes are likewise similar to 5-year results of the TAG trial [11], including similar overall survival (73\% at 55 months current study versus $68 \%$ at 60 months TAG) and re-intervention rates (6.3\% current study versus $3.6 \% \mathrm{TAG}$ ). One potential explanation for the greater than $50 \%$ reduction in paraplegia rate observed in the present series versus the clinical trials may be our routine use of intraoperative neurophysiologic monitoring with both somatosensory and motor-evoked potentials [7]. This modality, particularly the use of motor-evoked potentials, allows near instantaneous detection of spinal cord ischemia without having to wait for the patient to emerge from anesthesia. This early detection leads to earlier treatment with blood pressure augmentation and cerebrospinal fluid drainage and likely better spinal cord (and patient) outcomes.

We have found no published or pending randomized trials comparing TEVAR and open surgical treatment for any disorder of the thoracic aorta, and randomized controlled trials are unlikely in the future [12]. Results with open repair of descending thoracic aneurysm in centers of excellence have recently been summarized $[5,13]$. Mean patient age in these studies has generally been about 65 years, with 15 to $20 \%$ of the cases being nonelective. Thirty-day in-hospital rates of death ranged between 3 and 15\%, with rates of paraplegia and stroke ranging from 0 to $5 \%$ and 1.5 to $3.5 \%$, respectively. Renal failure was reported to occur in 0 to $7.5 \%$ of cases. The 30-day data from the present study compare favorably with those for open repair in centers of excellence, albeit in an older patient population with a higher percentage of nonelective cases. As advanced age has been associated with adverse outcomes in open thoracic aneurysm repair and nonelective status essentially doubles operative risk [13], risk-adjusted 30-day results with TEVAR are likely superior to those with conventional repair even in centers of excellence. Further, it is likely that conventional repair would have been denied many of the patients undergoing TEVAR for their lethal aortic disease in the present study. Follow-up outcomes after TEVAR in the present study are similar to open repair [14] as well, including a low need for re-intervention and similar follow-up survival.

Several important limitations should be considered when interpreting the present findings. First, this was an observational study consisting of retrospective analysis of prospectively collected data and not a controlled clinical trial. Second, the follow-up remains midterm whereas long-term follow-up beyond five years post-FDA approval will be necessary before TEVAR can be definitively established as a reliable treatment modality for thoracic aneurysm [3]. Finally, the results presented represent outcomes in a high-volume academic center and may not be generalizable to smaller programs. Similar to conventional open descending aneurysm repair [15], results are likely to be superior in higher volume centers with the necessary infrastructure.

In summary, "on-label" use of TEVAR for aneurysms of the descending thoracic aorta yields excellent 30-day and late midterm outcomes, which compare favorably to those previously published for open repair in centers of excellence as well as those from prior TEVAR clinical trials. Given these favorable results, as well as the short hospital length of 
stay, early return to full activity, and potential to offer therapy to patients who are not candidates for conventional open repair, TEVAR appears to be emerging as the preferred treatment strategy for this pathology in patients with appropriate anatomy.

\section{References}

1. Makaroun MS, Dillavou ED, Kee ST, et al. Endovascular treatment of thoracic aortic aneurysms: results of the phase II multicenter trial of the GORE TAG thoracic endoprosthesis. J Vasc Surg. 2005; 41:1-9. [PubMed: 15696036]

2. Bavaria JE, Appoo JJ, Makaroun MS, Verter J, Yu Z-F, Mitchell RS. Gore TAG Investigators. Endovascular stent grafting versus open surgical repair of descending thoracic aortic aneurysms in low-risk patients: a multicenter comparative trial. J Thorac Cardiovasc Surg. 2007; 133:369-77. [PubMed: 17258566]

3. Hughes GC, Daneshmand MA, Swaminathan M, et al. "Real world" thoracic endografting: results with the Gore TAG device 2 years after U.S. FDA approval. Ann Thorac Surg. 2008; 86:1530-8. [PubMed: 19049744]

4. Hughes GC, Nienaber JJ, Bush EL, Daneshmand MA, McCann RL. Use of custom dacron branch grafts for "hybrid" aortic debranching during endovascular repair of thoracic and thoracoabdominal aortic aneurysms. J Thorac Cardiovasc Surg. 2008; 136:21-8. [PubMed: 18603048]

5. Svensson LG, Kouchoukos NT, Miller DC, et al. Expert consensus document on the treatment of descending thoracic aortic disease using endovascular stent-grafts. Ann Thorac Surg. 2008; 85(1 suppl):S1-41. [PubMed: 18083364]

6. Cooper DG, Walsh SR, Sadat U, et al. Treating the thoracic aorta in Marfan syndrome: surgery or TEVAR? J Endovasc Ther. 2009; 16:60-70. [PubMed: 19281290]

7. Husain AH, Swaminathan M, McCann RL, Hughes GC. Neurophysiologic intraoperative monitoring during endovascular stent graft repair of the descending thoracic aorta. J Clin Neurophysiol. 2007; 24:328-35. [PubMed: 17938601]

8. Chaikof EL, Blankensteijn JD, Harris PL, et al. Reporting standards for endovascular aortic aneurysm repair. J Vasc Surg. 2002; 35:1048-60. [PubMed: 12021727]

9. Cheung AT, Pochettino A, McGarvey ML, et al. Strategies to manage paraplegia risk after endovascular stent repair of descending thoracic aortic aneurysms. Ann Thorac Surg. 2005; 80:1280-9. [PubMed: 16181855]

10. Parsa CP, Daneshmand MA, Lima B, Balsara K, McCann RL, Hughes GC. Utility of remote wireless pressure sensing for endoleak detection after endovascular thoracic aneurysm repair. Ann Thorac Surg. 2010; 89:446-52. [PubMed: 20103319]

11. Makaroun MS, Dillavou ED, Wheatley GH, Cambria RP. Gore TAG Investigators. Five-year results of endovascular treatment with the Gore TAG device compared with open repair of thoracic aortic aneurysms. J Vasc Surg. 2008; 47:912-8. [PubMed: 18353605]

12. Abraha I, Romagnoli C, Montedori A, Cirocchi R. Thoracic stent graft versus surgery for thoracic aneurysm. Cochrane Database Syst Rev. 2009; 1:CD006796. [PubMed: 19160302]

13. Black JH III, Cambria RP. Current results of open surgical repair of descending thoracic aortic aneurysms. J Vasc Surg. 2006; 43(suppl A):6A-11A.

14. Stone DH, Brewster DC, Kwolek CJ, et al. Stent-graft versus open-surgical repair of the thoracic aorta: mid-term results. J Vasc Surg. 2006; 44:1188-97. [PubMed: 17145420]

15. Schermerhorn ML, Giles KA, Hamdan AD, Dalhberg SE, Hagberg R, Pomposelli F. Populationbased outcomes of open descending thoracic aortic aneurysm repair. J Vasc Surg. 2008; 48:821-7. [PubMed: 18586435] 


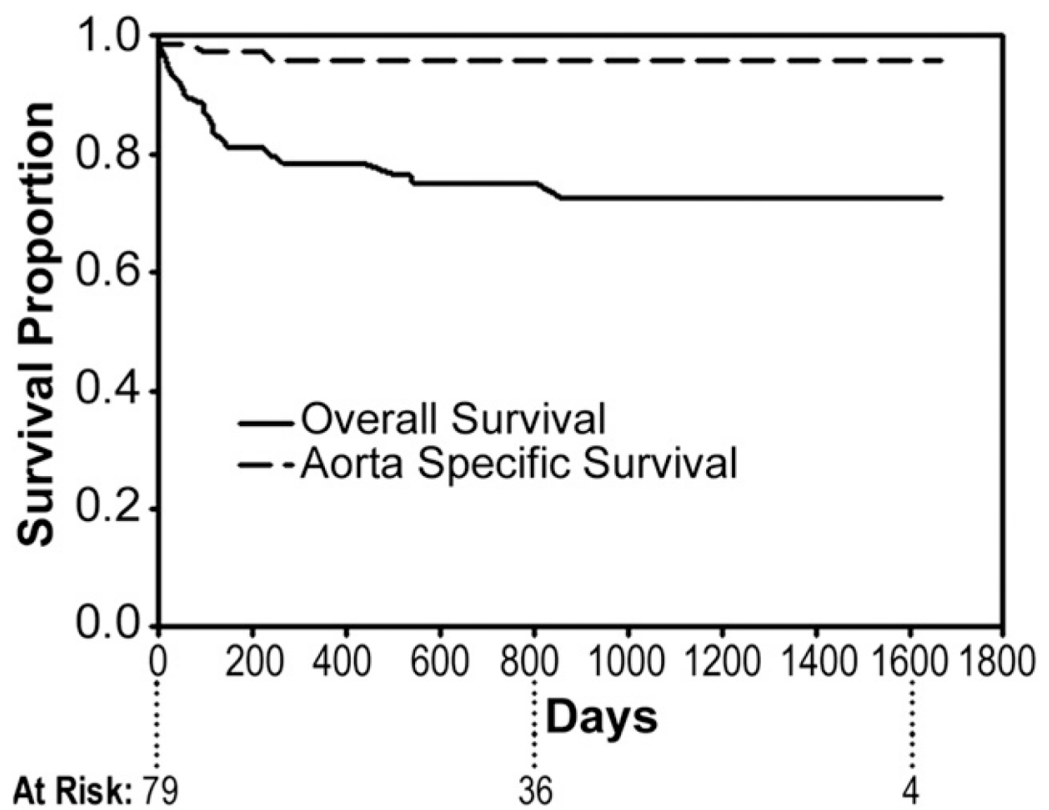

Fig 1.

Actuarial (Kaplan-Meier) overall (solid line) and aorta-specific (dashed line) survival at 55 months post-endovascular repair. 


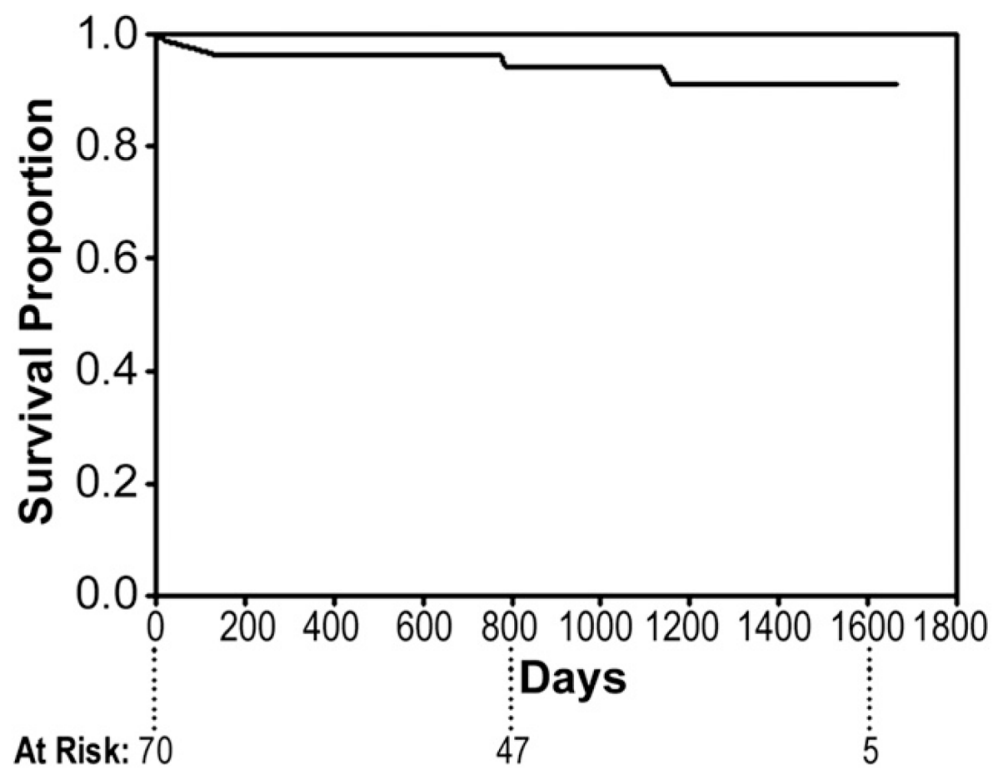

Fig 2.

Actuarial (Kaplan-Meier) freedom from late (>30 days) re-intervention at 55 months postendovascular repair. 
Table 1

Patient Demographics

\begin{tabular}{lc}
\hline Variable & No. $(\%)$ \\
\hline Median age & $73 \pm 14$ years \\
Female & $35(44 \%)$ \\
Hypertension & $69(87 \%)$ \\
Diabetes & $21(27 \%)$ \\
Coronary artery disease & $38(48 \%)$ \\
COPD & $43(54 \%)$ \\
Chronic renal insufficiency (baseline $\mathrm{Cr} \geq 1.5)$ & $22(28 \%)$ \\
Peripheral vascular disease & $43(54 \%)$ \\
Prior aortic surgery & $32(41 \%)$ \\
\hline
\end{tabular}

$\mathrm{COPD}=$ chronic obstructive pulmonary disease. 
Table 2

Length of Aortic Coverage by the Endograft(s)

\begin{tabular}{ll}
\hline Extent of Aortic "Pavement" (Types A-C) & No. (\%) \\
\hline Type A: Left subclavian artery to T6 & $29(37 \%)$ \\
Type B: Midthoracic aorta to celiac axis & $22(28 \%)$ \\
Type C: Left subclavian artery to celiac axis & $28(35 \%)$ \\
\hline
\end{tabular}

